\title{
The role of imaging in the initial work-up of paediatric renal tumours
}

Authors:

Dr Tom Watson MBChB ${ }^{1}$, Consultant Radiologist

Dr Minou Oostveen $\mathrm{MD}^{2}$, Clinical fellow in Oncology

Dr Harriet Rogers $\mathrm{PhD}^{3}$, Research Fellow

Professor Kathy Pritchard-Jones PhD, Professor of Paediatric Oncology ${ }^{2,4}$

Dr Øystein Olsen $\mathrm{PhD}^{1}$, Consultant Radiologist

1. Department of Paediatric Radiology, Great Ormond Street Hospital NHS Foundation Trust, London, UK

2. Department of Paediatric Haematology and Oncology, Great Ormond Street Hospital NHS Foundation Trust, London, UK

3. Centre for Medical Imaging, University College London, London, UK

4. UCL Great Ormond Street Institute of Child Health, University College London, London, UK

Corresponding author:

Dr Tom Watson

Department of Paediatric Radiology, Great Ormond Street Hospital for Children NHS Foundation Trust, Great Ormond Street, London, Wc1N 3JH, UK

Tom.watson@gosh.nhs.uk 


\section{$\underline{\text { Search strategy and selection criteria }}$}

Data for this review were identified from searches of MEDLINE, Pubmed, and references from relevant articles using the search terms; children or infants or paediatric or pediatric, and Wilms tumour or clear cell sarcoma or rhabdoid tumour or cystic partially differentiated nephroblastoma or cystic nephroma or multiloculated cystic nephroma or mesoblastic nephroma, and MRI or diffusion weighted imaging or CT or Positron emission tomography or ultrasound. Abstracts and meeting reports were not included. Articles published in English between January 2000 and April 2019 were included.

\section{$\underline{\text { Summary }}$}

Imaging plays a key role in the assessment of paediatric renal tumours, especially where the initial treatment approach is to proceed to standardised chemotherapy without histological confirmation. In Europe according to the SIOP guidelines, core needle biopsy is not routinely performed unless the child is older than 10 years. Between 6 months and 9 years, the child is treated with a standard regimen of pre-operative chemotherapy unless there concerns for nonWilms tumour pathology. Atypical imaging findings may therefore stratify a child into a different treatment protocol and may prompt the need for pre-treatment histology. This review details the latest protocols and techniques used in the assessment of paediatric renal tumours. Important imaging findings are considered especially those features which might prompt the need for a pre-treatment biopsy. Local radiology practices vary, both MRI and $\mathrm{CT}$ are widely used as a routine imaging test for the assessment of paediatric renal tumours in Europe. Advances in imaging technology and MRI sequences are facilitating the development of new techniques which may increase the utility of imaging in terms of predicting tumour histology and clinical behaviour. Several of these techniques are considered here. 


\section{$\underline{\text { Key points }}$}

1. Routine core needle biopsy of a renal mass is no longer recommended by UK and European guidelines for children between 6 months and 10 years with typical clinical and radiological features of a Wilms tumour

2. Ultrasound should be the first imaging modality for investigation of any suspected abdominal mass in a child

3. MRI or CT can be used interchangeably for the assessment of a known renal mass. MRI is recommended in the latest UMBRELLA research trial.

4. It is important to know the imaging features of a renal mass which suggest a non-Wilms tumour

5. Imaging cannot yet differentiate different tumour types or Wilms tumour stage but there are interesting areas of imaging research which may advance this area of imaging in the future

\section{$\underline{\text { Introduction }}$}

The diagnosis and treatment pathways for childhood renal tumours differ according to geography. In North America, the Childrens Oncology Group (COG) argues for upfront nephrectomy to obtain a definitive histological diagnosis and thus stratify treatment based on a 'chemo-naïve' tumour. Pre-operative chemotherapy is preserved for complex tumours ${ }^{1}$. The International Society of Paediatric Oncology Renal Tumours Study Group (SIOPRTSG), largely followed in Europe, argues in favour of pre-operative chemotherapy to downstage the tumour, reduce the risk of rupture and stratify post-operative treatment based on the histological response, as well as the tumour stage ${ }^{2}$. For a long time, SIOP has advocated for the use of standard chemotherapy regimens pre-operatively without histological confirmation in the form of a biopsy. All children aged over 6 months and with typical clinical findings for Wilms are treated according to this approach, since $90 \%$ of childhood renal tumours beyond early infancy are Wilms tumours. Biopsy is reserved for cases with clinical diagnostic uncertainty or older ages where the relative likelihood of a nonWilms tumour diagnosis starts to increase. The subjective nature of deciding when imaging appearances are 'typical' for Wilms tumour probably underlies the variation in reported 
biopsy rates of between $8-26 \%$ between different countries and European regions ${ }^{3}$. When the UK and Irish Children's Cancer and Leukaemia Group (CCLG) joined the SIOP-RTSG WT 2001 trial and study, they continued their national practice of performing a percutaneous biopsy (PCNB) prior to commencing chemotherapy in nearly all (97\%) of children with a renal tumour with the aim to prevent children with non-Wilms Tumour (non-WT) being administered unnecessary or suboptimal pre-operative chemotherapy. Recently the CCLG Renal Tumours Group have reviewed the diagnostic accuracy of paediatric renal tumour biopsies of children from the UK treated according the SIOP WT 2001 guidelines ${ }^{4}$. It was concluded that for children aged 6 months to 10 years where Wilms Tumour (WT) is the most likely diagnosis, the risk of biopsy outweighs the clinical benefits. In this age group a biopsy result rarely leads to a significant change in management from the standard SIOP approach. This has led to a change in the CCLG guidelines and biopsy is now reserved for cases where there are clinical or radiographic features to suggest a non-WT. In this context, imaging contributes a significant part of the pre-operative assessment. In particular, imaging needs to highlight cases where the radiological uncertainty is sufficient to warrant preoperative histology.

The SIOP Renal Tumour Study Group (RTSG) has developed a new protocol for the diagnosis and treatment of childhood renal tumours, the UMBRELLA SIOP-RTSG $2016^{2}$. One specific research question that involves imaging is the assessment of the independent prognostic value of the volume of the blastemal component of Wilms tumour that survives preoperative chemotherapy ${ }^{2}$. Imaging has an advantage over biopsy in this regard because the whole tumour volume can be evaluated. Currently there is scant evidence that this can be achieved using clinically validated imaging techniques.

In this paper, we review the current European recommendations for abdominal imaging in paediatric renal tumours. We discuss the necessary parameters for optimisation of MRI protocols and focus on key cross-sectional imaging features which, if present, may prompt consideration for core needle biopsy. There are a number of advanced techniques aimed at assessing tumour biology, in the case of Wilms tumour to assess the degree of anaplasia, degree of necrosis, and the blastemal content. A few of these techniques are available in clinical practice but in all cases, clear evidence is lacking for their efficacy. We will briefly 
summarise some of these techniques which may prove to be interesting areas for future research.

\section{$\underline{\text { Renal tumours in children }}$}

Primary malignant tumours of the kidney generally account for 4-7\% of cancers diagnosed in children under the age of 15 years in western industrialised countries. Wilms tumour or nephroblastoma is by far the most frequent histology accounting for $87 \%$ of tumours ${ }^{5}$. Between the ages of 1-9 years $92-95 \%$ of all renal malignant tumours are Wilms tumour but this figure drops to $81-89 \%$ in children $<1$ years of age and $54-67 \%$ for children $10-14$ years of age ${ }^{4,6,7}$. The most common non-WT in infants include Congenital Mesoblastic Nephroma $(\mathrm{CMN})$ and malignant rhabdoid tumour of the kidney (MRTK), whereas in adolescence, renal cell carcinoma (RCC) is more predominant. Clear cell sarcoma of the kidney (CCSK) has a similar age distribution to WT. Other malignant non-WT kidney tumours include soft tissue sarcomas/PNET, neuroblastoma of the kidney, non-Hodgkin lymphoma (primary site in the kidney), and teratoma. Other non-malignant renal tumours include cystic partially differentiated nephroblastoma, cystic nephroma and other rare entities.

Congenital mesoblastic nephroma CMN only accounts for 3-4\% of all paediatric renal tumours but is the most common renal neoplasm in the first 3 months of life ${ }^{8,9}$. In the UK $75 \%$ of all CMNs are diagnosed under the age of 6 months $^{8}$. Outcomes for patients with CMN are generally excellent when treated with nephrectomy only, with overall survival rates of about $95 \%$. The cellular subtype is more aggressive and these tumours may recur locally and may have metastatic potential ${ }^{9}$. A debate is currently ongoing whether patients with stage cellular III CMN would benefit from adjuvant chemotherapy. MRTK present a group of highly malignant childhood tumours with an early onset and median age at diagnosis of 1018 months. Advanced disease (stage III - IV) is reported in about two-thirds of cases at diagnosis. Even with a combination of very intensive chemotherapy and radiotherapy prognosis remains poor ${ }^{10,11}$. RCC account for $2-6 \%$ of paediatric malignant renal tumours. It is a highly chemotherapy and radiotherapy resistant disease. The majority of paediatric RCC are localized diseases, which can be cured with surgical tumour resection alone (survival rate of approximately $90 \%)^{12,13}$. For children with metastatic disease first line adjuvant therapy with the multi-targeted tyrosine kinase inhibitor Sunitinib is currently recommended. CCSK represents the second most common paediatric renal tumour after WT. It is classified as a 
'high risk' renal tumour, with metastatic potential to bone, and brain in addition to the lung and liver sites more typical of WT. With current intensive multi-agent chemotherapy regimens and radiotherapy 5-year overall survival ranges from $75-90 \%{ }^{14}$.

\section{Imaging of paediatric tumours}

To recommend a specific imaging protocol for any condition is challenging because of rapid advances in imaging technology and variation in local equipment and practice. An example MRI tumour protocol used at Great Ormond Street Hospital, London and recommended for use at UK centres is described in table 1.

It should be noted at the outset that ultrasound should be the initial modality in any suspected abdominal mass in a child ${ }^{15}$. MRI or CT are employed as the primary cross-sectional modalities once the initial diagnosis of a mass has been made by ultrasound. Ultrasound allows localisation of the lesion according to organ of origin (renal, hepatic,supra-renal etc) and guides the choice of cross-sectional modality.

MRI

According to the UMBRELLA protocol, MRI is considered the primary imaging modality for assessment of renal tumours. MRI often requires sedation or general anaesthetic in the younger age group, mainly because of the scan time and also because of the requirement for controlled breathing or breath holding. It can be difficult to arrange as an urgent investigation in many centres. This may be due to a number of factors such as lack of suitable scanners, expense, and limited availability of paediatric anaesthetists to provide safe sedation. MRI is also more expensive than CT in most countries, especially if sedation or anaesthesia is routinely required ${ }^{2}$. For these reasons and others, CT can be used interchangeably with MRI for diagnostic purposes.

The MRI field strength should be 1.0 Tesla as a minimum and realistically, it should be $1.5 \mathrm{~T}$ or higher. Recommended MRI sequences include: 
- Short Tau Inversion Recovery (STIR) sequences in the coronal and axial planes. These are fluid sensitive large field of view sequences which allow an overview of the abdomen. These may be replaced by other fat-suppressed T2-weighted spin echo sequences

- T2-weighted sequence without fat saturation in the axial plane. This is a high resolution sequence which should cover the whole of the tumour and both kidneys. It can be substituted for a volumetric T2 weighted spin echo sequence where available which allows for multiplanar reconstruction .

- Diffusion weighted images (DWI) acquired in the axial plane can be performed with the child freely breathing. Sequences are acquired with different strengths of gradient applied. These gradients are referred to as: b-values. Higher b-values increase the diffusion effect. These images currently provide a qualitative assessment of tumour cellularity.

- Administration of gadolinium is not mandatory. If performed, pre- and post- contrast sequences are necessary. Ideally these should be short sequences performed with a breath-hold. Post-contrast images should be taken in the arterial and portal venous phase if possible.

Volumetric T2 weighted sequences are useful for anatomical delineation of the tumour. As well as multiplanar reconstruction, they provide high resolution vascular detail (Fig. 1) ${ }^{16}$. DWI sequences can provide a qualitative and quantitative assessment of tissue, but to date, there is little literature which has proven a link between apparent diffusion coefficient (ADC) values and tumour subtype in terms of blastemal or epithelial content and degree of anaplasia. ADC values may vary according to the scanner field strength and between different manufacturers ${ }^{17}$. A very low ADC value (tumours which appear bright on higher b-value DWI sequences and very dark on the associated ADC map) implies increased cellularity, but this not necessarily correlated with tumour subtype ${ }^{18}$. In follow-up imaging, the ADC value can be used as an assessment of chemotherapeutic effect, good interobserver variability has been demonstrated in terms of measurement of ADC values and a difference of more than 0.1 $\mathrm{x} 10^{-3} \mathrm{~mm}^{2} / \mathrm{s}$ is considered unlikely to be due to random error. Changes in ADC values following chemotherapy are often considerably larger than this ${ }^{18,19}$. For initial tumour staging, DWI is useful for detection of smaller lesions in both kidneys and also for detection 
of lymph node or distant metastases however all patients will have lymph node sampling at nephrectomy and therefore whilst useful to detect obvious lymph node metastases, their absence will not change surgical management.

CT

Although MRI is considered as the optimal imaging modality in the UMBRELLA research study, it is not necessarily considered so by all centres/countries for clinical assessment. As previously stated CT is the modality of choice in North America. Many other centres will also prefer to use CT because it is easier to obtain and much easier to standardise in terms of technique. The main concern about the use of CT has always been the radiation burden imparted by the study ${ }^{20}$. Whilst this is an important consideration for long term follow-up, MRI is not a risk-free long term strategy as evidenced by the recent concerns about the neurocognitive effects of general anaesthetic and the controversial issue of gadolinium accumulation in the deep white matter of the brain ${ }^{21,22}$. It has been shown that omitting the pelvis in follow-up CT studies can reduce the effective dose of the study by up to $45 \%$ without significant reduction in relapse detection ${ }^{23,24}$.

A single phase acquisition of the abdomen and pelvis with intra-venous contrast in the portal venous phase is the commonly accepted protocol for assessment of renal tumours in children $^{25,26}$. The tumour will typically enhance to a lesser degree than the surrounding renal parenchyma and the portal venous phase is usually sufficient to identify the main renal vessels and the IVC. Multi-phase CT can provide unenhanced, arterial and late phase contrast enhancement but it is questionable what additional information these acquisitions provide when balanced against the increase in radiation burden. There is some evidence that an unenhanced protocol is helpful to detect calcifications within the lesion. This is an unusual finding in Wilms tumour and would be more indicative of a non-Wilms tumour such as a renal cell carcinoma ${ }^{27,28}$. CT has been shown to be comparable to MRI in terms of capsular penetration and presence/extent of IVC thrombus. In terms of lymph node detection, CT was shown by Servaes et al ${ }^{26}$ to be better than MRI, remembering that absence of lymph node metastases on imaging does not remove the need for surgical lymph node sampling. MRI was slightly more accurate for detection of synchronous tumours in this group. This study from the Children's Oncology Group makes the conclusion that either test is reasonable for initial tumour staging. 
CT remains the standard of care for assessment of lung metastases. Therefore an assessment of the chest can be performed at the same time as the CT of the abdomen and pelvis which confers a significant time advantage for CT. This will also allow a reduction in the radiation dose but only if the imaging of the chest, abdomen, and pelvis is acquired in a single scan.

In the SIOP protocol, a pre-operative, post-chemotherapy imaging study is performed in addition to the diagnostic scan. This provides important information about the surgical resectability of the tumour for the operating surgeon and allows accurate assessment of the residual tumour volume. Several authors have shown that this second CT scan can demonstrate changes due to chemotherapy beyond that of simple volume reduction ${ }^{29,30}$. As with MRI, these CT features do not appear to be able to distinguish between histopathological tumour subtypes.

\section{Imaging features}

In this section, we discuss the salient imaging features that the radiologist should evaluate as part of image analysis. CT or MRI can be used to perform this evaluation where necessaary, we have indicated which of the two modalities may be preferred. As well a general review of the tumour characteristics such as location, size, and signal/density; paediatric renal tumour imaging review should include an assessment for several important features.

\section{Capsular rupture}

Intra-peritoneal rupture of the tumour upstages it to Stage 3 but can often occur on a microscopic level and thus accurate detection with imaging is limited to the most advanced cases. Retroperitoneal rupture may be suspected on imaging, but the final diagnosis should rest on findings at surgery and post-resection histology - together these are considered the gold standard for assessment of rupture. CT has been studied more extensively in this regard but both CT and MRI have similar specificity of $85-90 \%$ based on small numbers of cases ${ }^{26}$, 31. Both modalities are able to produce volumetric datasets which can be reconstructed in any desirable plane to improve interpretation (Fig 2). Imaging features associated with rupture include: 1)ascites beyond the cul-de-sac 2)extra-capsular retroperitoneal fluid 3)peritoneal deposits (Fig. 2). A small volume of ascites (especially if limited to the pelvis), the presence of intratumoral haemorrhage, and subcapsular haemorrhage/fluid are not considered indicative of intraperitoneal rupture on imaging. It is important to recognise the distinction 
between rupture into the peritoneal space and extra-capsular, but retroperitoneal rupture. Extra-capsular rupture means tumour extension beyond the renal capsule, but limited to the retroperitoneum. This is usually apparent on imaging due to disruption of the smooth tumour margin and irregular tumour extending into the peri-renal fat and the retro-peritoneum. Extra-capsular rupture is important to recognise on imaging but does not always imply a change in the tumour stage ${ }^{32}$.

Intravascular extension

Approximately $4-10 \%$ of Wilms tumours will present with intravascular extenstion, and 1$3 \%$ with extension into the right atrium ${ }^{25}$. This has implications for surgical planning as surgery can be complex and is associated with significant operative mortality ${ }^{33}$. Both CT and MRI are effective for detection of thrombus ${ }^{34,35}$. MRI is considered the preferred method for intravascular extension ${ }^{26}$, but it does not follow that if IVC thrombus is detected on CT then MRI is required for assessment. MRI does not require intravenous contrast for thrombus detection because the absence of flow voids on T2 weighted imaging can also show the thrombus effectively (Fig. 3), but intravenous contrast may help to distinguish malignant thrombus from bland thrombus as the former should enhance. It should be noted that both types of thrombus can co-exist in the same patient with bland thrombus often arising downstream of the obstructing malignant thrombus ${ }^{36}$. DWI can also be helpful in distinction of bland and malignant thrombus, but caution should be exercised as the presence of haemoglobin can affect local magnetic fields. This results in artefactual signal within the thrombus which cannot be relied upon for assessment.

Bilaterality

Approximately 5-8\% of Wilms tumours are bilateral. In many patients with bilateral tumours, there is an underlying syndrome or genetic mutation which predisposes the child ${ }^{37}$. Amongst these, the most common are; Beckwith -Wiedemann syndrome and the WT1 mutation- associated syndromes. Nearly all (>90\%) cases are associated with nephrogenic rests. These are embryological remnants of the metanephros and are either located on the peripheral cortex (perilobar) or within the renal substance itself (intralobar) ${ }^{38}$. They occur sporadically in normal children and the natural history is unknown. What is is clear though, is that nephroblastoma and nephrogenic rests frequently co-exist and the presence of multiple nephrogenic rests is common in cases of bilateral nephroblastoma. Distinguishing malignant tumours from rests on imaging is notoriously difficult, especially in cases where the 
nephrogenic rests are bilateral, large, and diffuse, a condition known as nephroblastomatosis (Fig. 4). MRI is considered to be more effective in the detection of synchronous lesions and is the preferred imaging modality in both the SIOP and COG for evaluation of renal lesions in children with known renal tumour predisposition ${ }^{26}$. Distinction of rests from tumour on imaging is difficult. The evidence base to support the radiologist is limited and often derived from expert opinion and observational work without a robust gold standard (i.e. histology). Nephrogenic rests tend to be smaller $(<2 \mathrm{~cm})$ and ovoid or lenticular, with uniform signal intensity and lower enhancement than background cortex. Wilms tumours tend to be spherical and larger, however there is considerable overlap in these appearances and caution should be exercised when assigning a histological label to small renal masses in the contralateral kidney in cases of proven Wilms tumour. In our experience, the most useful sequences for detection of additional lesions, in no particular order, are: DWI, arterial postcontrast phase and the volumetric 3D T2. The contralateral kidney and any uninvolved poles of the affected kidney should be scrutinized on these sequences for the presence of additional masses. The presence and location of additional and bilateral masses has important implications for the pre-operative chemotherapy regimen and for the feasibility of nephronsparing surgery ${ }^{39}$.

Imaging features which should prompt biopsy:

The SIOP protocol lists a number of radiological features that, if present, should prompt consideration of biopsy. In general, these features suggest an alternative diagnosis, which might require completely different management and is thus important to establish at diagnosis if possible. This is not an exhaustive list and the final decision regarding biopsy should be made by clinical discussion usually within a multi-disciplinary team.

In most general radiology textbooks, nephroblastoma is said to 'displace' adjacent structures including the large abdominal vessels, this is because it is confined by the strong fibrous perirenal capsule ${ }^{40}$. In contrast, neuroblastoma, which is derived from neural crest cells located most commonly in the adrenal/suprarenal glands, is not restricted by a capsule. Vascular encasement is therefore a common feature of suprarenal neuroblastoma ${ }^{40}$. If vascular encasement is present when evaluating a presumed renal mass, an unencapsulated tumour such as neuroblastoma should be suspected (Fig. 5), though a ruptured Wilms tumour could behave in a similar fashion. Other imaging features which suggest an unencapsulated tumour 
include: a large retroperitoneal and extra-renal mass, invasion of adjacent structures in the retroperitoneum, in particular the psoas muscle.

Nephroblastoma most commonly metastases to the lungs. Although much less frequent, the next most common site is the liver ${ }^{41}$. The presence of other sites of metastatic disease is unusual. In particular, bone metastases are more common in CCSK ${ }^{14}$ and brain metastases or synchronous primary brain tumours are more often seen in $\mathrm{MRTK}^{10}$. Unfortunately, there is little that has been shown to reliably distinguish the primary renal tumour in each of these entities from nephroblastoma.

In children under the age of two, the presence of pulmonary metastases is regarded as unusual for Wilms tumour. Pulmonary metastatic disease at this young age is more often a feature of non-Wilms tumour and may prompt a biopsy prior to chemotherapy ${ }^{41}$. However, as stated previously, caution should be exercised here because core needle biopsy has been shown to be inaccurate for distinguishing rare tumour subtypes ${ }^{4}$.

Cystic Partially Differentiated Nephroblastoma (CPDN) can encompass a number of diagnoses on imaging and should be considered as a spectrum of disorders extending from the benign cystic nephroma to the malignant entity of cystic nephroblastoma. It is considered to be a 'low risk' renal tumour ${ }^{42}$. On imaging, these lesions should be almost entirely cystic. Any enhancing solid components should be limited to thin septae (Fig. 6). The presence of larger solid elements suggests a malignant tumour such as a nephroblastoma with cystic degeneration. A characteristic feature of CPDN on imaging is extension of the tumour into the collecting system ${ }^{43}$.

\section{$\underline{\text { Advanced Imaging in Wilms tumours }}$}

In this section, we review techniques which are currently being used in research studies with the aim of enhancing the capabilities of imaging in oncology. Some techniques have been specifically used in paediatric renal tumours; others are being used more generally in oncology imaging. It is by no means an exhaustive list. Most of these techniques are not in routine clinical use and it will require resources to provide these in a clinical environment both in terms of software and hardware and in terms of training for clinical staff to operate and interpret. As things stand at time of writing, an ultrasound for initial investigation followed by CT or MRI for definitive imaging remains the state-of-the-art from a clinician's perspective. 


\section{$\underline{\text { MRI - Diffusion }}$}

MRI can provide a variety of images with different contrasts. This aids the identification of tumours, and assists with the delineation of boundaries and margins. This form of imaging can be known as qualitative; it relies on visual inspection of the produced images. An additional benefit of MRI is that one can use the quantitative information.

Diffusion weighted imaging (DWI) is frequently acquired for Wilms tumours, and from this the ADC can be calculated. ADC has been found to be related to cell count in a range of abdominal paediatric tumours (a subset of which were Wilms tumours), with a decrease in ADC being significantly related to an increase in cell count ${ }^{19}$. This relationship has assisted with separating benign abdominal tumours from malignant tumours, as benign tumours have reduced cellularity and thus have a higher $\mathrm{ADC}^{44}$.

ADC can also be used to assess chemotherapy response. Tumours with large volumes of necrotic tissue may not reduce significantly in size following chemotherapy. This means that traditional volume assessments may be unreliable to assess treatment efficacy ${ }^{45}$. By comparing distributions of ADC values pre- and post-chemotherapy it was shown by McDonald et al that these distributions changed and that median ADC values increased post treatment. In addition to this, ADC distributions have been used to distinguish Wilms tumour subtypes; it was shown that the higher risk blastemal subtype had significantly lower ADC values compared to stromal, regressive and mixed subtypes ${ }^{46}$. Currently, subtypes can only be assessed via histological analysis and thus this non-invasive method has the potential to provide important information which may help guide treatment. These feasibility studies demonstrate that $\mathrm{ADC}$ distributions have the potential to be a non-invasive biomarker for monitoring treatment response, however we should keep in mind the limitations discussed earlier in this paper regarding the reliability and repeatability of ADC quantification.

Intravoxel incoherent motion (IVIM) is a multiple b-value DWI technique which uses a different algorithm from conventional DWI sequences. It can separate the effect of the microcirculation and the true diffusion signal derived from the movement of extracellular water allowing separate analysis of perfusion and diffusion. Currently, this is limited to the 
research environment but in a small paediatric trial, Meeus et al demonstrated that IVIM techniques can distinguish malignant tumours from benign entities; they were unable to distinguish tumour types in their cohort ${ }^{47}$.

Gadolinium-enhanced sequences are commonplace in renal tumour assessment. The resulting images will display regions which have limited/no vascularity (low signal) and therefore may be necrotic and areas which have a good vascular supply (bright signal) and therefore may be viable tumour. Gadolinium has side effects, can raise examination costs and is contraindicated in those with renal failure. An alternative MRI technique would be useful. Advances in imaging have suggested that it is possible to generate a threshold to quantify necrotic tissue without using gadolinium ${ }^{48}$. Through combining ADC and $\mathrm{T}_{1}$-weighted sequences, images can be generated which resemble gadolinium-enhanced images, and the volume of necrosis can be quantified which is informative of chemotherapy-induced changes. (Fig. 7). Again, this remains a research technique at present, but an accurate MRI without the need for gadolinium would be highly desirable.

CT - Texture analysis

CT texture analysis is a new technique which analyses the greyscale values of individuals pixels within a defined volume, it requries additional software to perform the analysis. It is not yet a clinical technique in oncology imaging for adults or children but early studies are positive with regards to the feasibility of distinguishing malignant and non-malignant lesions ${ }^{49}$.

Other modalities

Nuclear medicine

Positron Emission Tomography (PET) can be paired with CT and has been shown to detect Wilms tumour and differentiate this from benign entities ${ }^{50-52}$. It does not form part of the standard imaging work-up for paediatric renal tumours, in part because of the high radiation dose but with PET-MRI becoming more commonplace and acceptable in paediatric 
oncology ${ }^{53}$, the additional information provided by functional imaging may become more desirable.

Ultrasound

Ultrasound should be the first imaging for any child with a suspected abdominal mass ${ }^{15}$. It is can be employed as a screening tool in those children with pre-disposition syndromes such as Beckwith-Wiedemann syndrome. Advanced ultrasound techniques such as contrastenhancement using an intravenous injection of microbubbles may prove useful for characterisation of suspected renal masses. Contrast-enhanced ultrasound has been used in the UK in adults for more than a decade and is described for distinguishing malignant renal tumours from pseudotumours in adults ${ }^{54-56}$. The main advantage is that one can perform real-time imaging with quantitative analysis of dynamic contrast enhancement. Although advanced ultrasound techniques will not replace the volume acquisition and reproducibility of MRI, they may have a role in children with pre-disposition syndromes where abnormal renal echotexture can often raise concern for a malignancy. Qualitative and quantitative review of a contrast-enhanced ultrasound may help to distinguish normal renal tissue from a solid tumour and thus stratify patients to routine follow-up or further investigation.

\section{Conclusions:}

A 'no-biopsy' approach to the management of paediatric renal masses places additional emphasis on the capabilities of pre-operative imaging for accurate diagnosis and risk stratification to ensure appropriate pre-operative care. In terms of imaging, high-quality MRI or CT can demonstrate important features to assist in predicting histological diagnosis in combination with clinical features and informing the choice of initial treatment approach (i.e. empirical pre-operative chemotherapy, biopsy or immediate nephrectomy). In the future, there may be sequences and techniques which enable characterization of tumour in terms of histological subtype and eliminate the need for gadolinium. However, these are not sufficiently reliable for routine clinical practice at this time.

\section{Declaration of interests:}

None declared 
TW - Lead author, wrote the manuscript and prepared the article for publication MO - Contributed to sections of the manuscript and reviewed the final draft HR - Contributed to sections of the manuscript and reviewed the final draft KPJ - Contributed to concept for the manuscript, reviewed and edited early versions and reviewed the final draft

$\mathrm{OO}$ - Reviewed and edited early versions of the manuscript and reviewed the final draft 


\section{$\underline{\text { References }}$}

1 Wilms Tumor and Other Childhood Kidney Tumors Treatment-for health professionals (PDQ®). PDQ Pediatric Treatment Editorial Board. PDQ Cancer Information Summaries. Natl Cancer Inst 2015. Retrieved from http://www.ncbi.nlm.nih.gov/pubmed/26389282 on September $1^{\text {st }} 2019$

2 Van Den Heuvel-Eibrink MM, Hol JA, Pritchard-Jones K, et al. Position Paper: Rationale for the treatment of Wilms tumour in the UMBRELLA SIOP-RTSG 2016 protocol. Nature Reviews Urology, 14(12), 743-752. https://doi.org/10.1038/nrurol.2017.163

3 Irtan S, Van Tinteren H, Graf N, et al. Evaluation of needle biopsy as a potential risk factor for local recurrence of Wilms tumour in the SIOP WT 2001 trial. Eur J Cancer 2019; 116: $13-20$.

4 Jackson TJ, Williams RD, Brok J, et al. The diagnostic accuracy and clinical utility of pediatric renal tumor biopsy: Report of the UK experience in the SIOP UK WT 2001 trial. Pediatr Blood Cancer 2019; 66(6): e27627.

5. Stiller C.A., Olshan A.F. Epidemiology of Renal Tumours of Childhood. In: PritchardJones K., Dome J. (eds) Renal Tumors of Childhood. Pediatric Oncology. 2014. Springer, Berlin, Heidelberg. DOI: https://doi.org/10.1007/978-3-662-44003-2_1

6 Pastore G, Znaor A, Spreafico F, et al. Malignant renal tumours incidence and survival in European children (1978-1997): Report from the Automated Childhood Cancer Information System project. Eur J Cancer 2006; 42(13), 2103-2114.

DOI:10.1016/j.ejca.2006.05.010.

7 Cancer Research UK, Public Health England. National Cancer Registration and Analysis Service Short Report: Chemotherapy, Radiotherapy and Surgical Tumour Resections in England: 2013-2014 (V2). 2017 DOI:10.1016/S1470-2045(05)70436-8. 
8 England RJ, Haider N, Vujanic GM, et al. Mesoblastic nephroma: A report of the United Kingdom children's cancer and leukaemia group (CCLG). Pediatr Blood Cancer 2011; 56(5), 744-748.. DOI:10.1002/pbc.22871.

9 Gooskens SL, Houwing ME, Vujanic GM, et al. Congenital mesoblastic nephroma 50 years after its recognition: A narrative review. Pediatr Blood Cancer 2017; 64: e26437 DOI:10.1002/pbc.26437.

10 Van den Heuvel-Eibrink MM, van Tinteren H, Rehorst H, et al. Malignant rhabdoid tumours of the kidney (MRTKs), registered on recent SIOP protocols from 1993 to 2005: A report of the SIOP renal tumour study group. Pediatr Blood Cancer 2011. 56(5), 733-737. DOI:10.1002/pbc.22922.

11 Brennan B, Stiller C, Bourdeaut F. Extracranial rhabdoid tumours: What we have learned so far and future directions. Lancet Oncol. 2013: 14(8) 329-336.

DOI:10.1016/S1470-2045(13)70088-3.

12 Spreafico F, Collini P, Terenziani M, Marchianò A, Piva L. Renal cell carcinoma in children and adolescents. Expert Rev. Anticancer Ther. 2010; 10(12): 1967-1978 DOI:10.1586/era.10.188.

13 Selle B, Furtwängler R, Graf N, Kaatsch P, Bruder E, Leuschner I. Population-based study of renal cell carcinoma in children in Germany, 1980-2005. Cancer 2006; 107(12), 2906-2914. DOI:10.1002/cncr.22346.

14 Gooskens SLM, Furtwängler R, Vujanic GM, Dome JS, Graf N, Van Den HeuvelEibrink MM. Clear cell sarcoma of the kidney: A review. Eur J Cancer 2012; 48(14), 22192226. DOI:10.1016/j.ejca.2012.04.009.

15 Saltzman AF, Carrasco A, Weinman J, Meyers ML, Cost NG. Initial Imaging for Pediatric Renal Tumors: An Opportunity for Improvement. J Urol 2018; 199(5), 1330-1336 DOI:10.1016/j.juro.2017.11.076. 
16 Dias SC, Olsen ØE. Isotropic 3-D T2-weighted spin-echo for abdominal and pelvic MRI in children. Pediatr Radiol 2012; 42(11) 1385-1390. DOI:10.1007/s00247-012-2395-1.

17 Littooij AS, Sebire NJ, Olsen ØE. Whole-tumor apparent diffusion coefficient measurements in nephroblastoma: Can it identify blastemal predominance? J Magn Reson Imaging 2017; 45(5), 1316-1324. DOI:10.1002/jmri.25506.

18 Littooij AS, Humphries PD, Olsen OE. Intra- and interobserver variability of whole tumour apparent diffusion coefficient measurements in nephroblastoma: a pilot study. Pediatr Radiol 2015; 45: 1651-60.

19 Humphries PD, Sebire NJ, Siegel MJ, Olsen OE, Cellularity T. Tumors in Pediatric Patients at Apparent Diffusion Coefficient and. Radiology 2007 245(3):848-54.

20 Pearce MS, Salotti JA, Little MP, et al. Radiation exposure from CT scans in childhood and subsequent risk of leukaemia and brain tumours: A retrospective cohort study. Lancet 2012; 380(9840) 499-505. DOI:10.1016/S0140-6736(12)60815-0.

21 Cauldwell C. Anesthesia risks associated with pediatric imaging. Pediatr Radiol 2011; 41:949-950 . DOI:10.1007/s00247-011-2160-х.

22 Kanda T, Ishii K, Kawaguchi H, Kitajima K, Takenaka D. High Signal Intensity in the Dentate Nucleus and Globus Pallidus on Unenhanced T1-weighted MR Images: Relationship with Increasing Cumulative Dose of a Gadolinium-based Contrast Material. Radiology 2014; 270(3) 834-841. DOI:10.1148/radiol.13131669.

23 Kaste SC, Brady SL, Yee B, et al. Is routine pelvic surveillance imaging necessary in patients with Wilms tumor? Cancer 2013; 119(1) 182-188. DOI:10.1002/cncr.27687.

24 Kan JH, Hwang M, Lowas SR, Hernanz-Schulman M. Impact of pelvic CT on staging, surveillance, and survival of pediatric patients with wilms tumor and hepatoblastoma. Am J Roentgenol 2011; 96:W515-W518. DOI:10.2214/AJR.10.5179.

25 Brisse HJ, Smets AM, Kaste SC, Owens CM. Imaging in unilateral Wilms tumour. In: 
Pediatric Radiology. 2008; 38(1): 18-29. DOI:10.1007/s00247-007-0677-9.

26 Servaes S, Khanna G, Naranjo A, et al. Comparison of diagnostic performance of CT and MRI for abdominal staging of pediatric renal tumors: a report from the Children's Oncology Group. Pediatr Radiol 2015; 45(2) 166-172. DOI:10.1007/s00247-014-3138-2.

27 Riccabona M. Imaging of renal tumours in infancy and childhood. Eur. Radiol. 2003; 13(Suppl 6): L116. DOI:10.1007/s00330-003-2001-X.

28 Lonergan GJ, Martínez-León MI, Agrons GA, Montemarano H, Suarez ES. Nephrogenic Rests, Nephroblastomatosis, and Associated Lesions of the Kidney. Radiographics 1998; 18(4) 947-968. DOI:10.1148/radiographics.18.4.9672980.

29 Olsen OE, Jeanes AC, Sebire NJ, et al. Changes in computed tomography features following preoperative chemotherapy for nephroblastoma: Relation to histopathological classification. Eur Radiol 2004; 14(6) 990-994. DOI:10.1007/s00330-003-2217-9.

30 Gow KW, Roberts IF, Jamieson DH, Bray H, Magee JF, Murphy JJ. Local staging of Wilms' tumor - Computerized tomography correlation with histological findings. J Pediatr Surg 2000; 35(5) 677-679. DOI:10.1053/jpsu.2000.5941.

31 Khanna G, Naranjo A, Hoffer F, et al. Detection of Preoperative Wilms Tumor Rupture with CT: A Report from the Children's Oncology Group. Radiology 2012; 66(2) 610-617. DOI:10.1148/radiol.12120670.

32 Le Rouzic MA, Mansuy L, Galloy MA, Champigneulle J, Bernier V, Chastagner P. Agreement between clinicoradiological signs at diagnosis and radiohistological analysis after neoadjuvant chemotherapy of suspected Wilms tumor rupture: Consequences on therapeutic choices. Pediatr Blood Cancer 2019; 66(6): e27674. DOI:10.1002/pbc.27674.

33 Lall A, Pritchard-Jones K, Walker J, et al. Wilms' tumor with intracaval thrombus in the UK Children's Cancer Study Group UKW3 trial. In: Journal of Pediatric Surgery. 2006; 41(2) 382-387. DOI:10.1016/j.jpedsurg.2005.11.016. 
34 Khanna G, Rosen N, Anderson JR, et al. Evaluation of diagnostic performance of CT for detection of tumor thrombus in children with Wilms tumor: A report from the Children's Oncology Group. Pediatr Blood Cancer 2012; 58(4) 551-555. DOI:10.1002/pbc.23222.

35 McDonald K, Duffy P, Chowdhury T, McHugh K. Added value of abdominal crosssectional imaging (CT or MRI) in staging of Wilms' tumours. Clin Radiol 2013; 68(1) 16-20. DOI:10.1016/j.crad.2012.05.006.

36 Kaufman LB, Yeh BM, Breiman RS, Joe BN, Qayyum A, Coakley F V. Inferior vena cava filling defects on CT and MRI. Am J Roentgenol 2005; 185(3) 717-726.

DOI:10.2214/ajr.185.3.01850717.

37 Charlton J, Irtan S, Bergeron C, Pritchard-Jones K. Bilateral Wilms tumour: a review of clinical and molecular features. Expert Rev Mol Med 2017; 19: e8.

38 Owens CM, Brisse HJ, Olsen ØE, Begent J, Smets AM. Bilateral disease and new trends in Wilms tumour. In: Pediatric Radiology. 2008; 38(1) 30-39. DOI:10.1007/s00247007-0681-0.

39 Murphy AJ, Davidoff AM. Bilateral Wilms Tumor: A Surgical Perspective. Child (Basel, Switzerland) 2018; 5(10): 134. DOI:10.3390/children5100134.

40 Dumba M, Jawad N, McHugh K. Neuroblastoma and nephroblastoma: a radiological review. Cancer imaging Off Publ Int Cancer Imaging Soc 2015; 15(1): 5.

41 Swinson S, McHugh K. Urogenital tumours in childhood. Cancer imaging Off Publ Int Cancer Imaging Soc 2011; 11 Spec No: S48-64.

42 Popov SD, Sebire NJ, Vujanic GM. Wilms' Tumour - Histology and Differential Diagnosis (pp 3-21). In: van den Heuvel-Eibrink MM, ed. Wilms Tumor. Codon Brisbane (AU), 2016. DOI:10.15586/codon.wt.2016.ch1. 
43 Silver IMF, Boag AH, Soboleski DA. Best cases from the AFIP: Multilocular cystic renal tumor: cystic nephroma. Radiographics 2008; 28(4) 1221-1227.

DOI:10.1148/rg.284075184.

44 Gawande RS, Gonzalez G, Messing S, Khurana A, Daldrup-Link HE. Role of diffusion-weighted imaging in differentiating benign and malignant pediatric abdominal tumors. Pediatr Radiol 2013; 43(7) 836-845. DOI:10.1007/s00247-013-2626-0.

45 McDonald K, Sebire NJ, Anderson J, Olsen ØE. Patterns of shift in ADC distributions in abdominal tumours during chemotherapy-feasibility study. Pediatr Radiol 2011; 41(1) 99106. DOI:10.1007/s00247-010-1741-4.

46 Hales PW, Olsen ØE, Sebire NJ, Pritchard-Jones K, Clark CA. A multi-Gaussian model for apparent diffusion coefficient histogram analysis of Wilms' tumour subtype and response to chemotherapy. NMR Biomed 2015; 28(8) 948-957. DOI:10.1002/nbm.3337.

47 Meeus EM, Zarinabad N, Manias KA, et al. Diffusion-weighted MRI and intravoxel incoherent motion model for diagnosis of pediatric solid abdominal tumors. J Magn Reson Imaging 2018; 47(6) 1475-1486. DOI:10.1002/jmri.25901.

48 Rogers HJ, Verhagen M V., Shelmerdine SC, Clark CA, Hales PW. An alternative approach to contrast-enhanced imaging: diffusion-weighted imaging and T1-weighted imaging identifies and quantifies necrosis in Wilms tumour. Eur Radiol 2018; 29(8) 4141 4149. DOI:10.1007/s00330-018-5907-z.

49 Cahalane AM, Kilcoyne A, Tabari A, McDermott S, Gee MS. Computed tomography texture features can discriminate benign from malignant lymphadenopathy in pediatric patients: a preliminary study. Pediatr Radiol 2019; 49(6) 737-745. DOI:10.1007/s00247-01904350-3.

50 Begent J, Sebire NJ, Levitt G, et al. Pilot study of F18-Fluorodeoxyglucose Positron Emission Tomography/computerised tomography in Wilms tumour: Correlation with conventional imaging, pathology and immunohistochemistry. Eur J Cancer 2011; 47(3) 389396. DOI:10.1016/j.ejca.2010.09.039. 
51 Hossain AKMM, Shulkin BL, Gelfand MJ, et al. FDG positron emission tomography/computed tomography studies of Wilms' tumor. Eur J Nucl Med Mol Imaging 2010; 37(7) 1300-1308. DOI:10.1007/s00259-010-1396-2.

52 Shulkin BL, Chang E, Strouse PJ, Bloom DA, Hutchinson RJ. PET FDG studies of wilms tumors. Am J Pediatr Hematol Oncol 1997; 19(4) 334-338. DOI:10.1097/00043426199707000-00012.

53 Pfluger T, Melzer HI, Mueller WP, et al. Diagnostic value of combined 18F-FDG PET/MRI for staging and restaging in paediatric oncology. Eur J Nucl Med Mol Imaging 2012; 39(11) 1745-1755. DOI:10.1007/s00259-012-2228-3.

54 Wei S-P, Xu C-L, Zhang Q, et al. Contrast-enhanced ultrasound for differentiating benign from malignant solid small renal masses: comparison with contrast-enhanced CT. Abdom Radiol (New York) 2017; 42: 2135-45.

55 Sanz E, Hevia V, Arias F, et al. Contrast-enhanced ultrasound (CEUS): an excellent tool in the follow-up of small renal masses treated with cryoablation. Curr Urol Rep 2015; 16: 469 .

56 Rubenthaler J, Negrao de Figueiredo G, Mueller-Peltzer K, Clevert DA. Evaluation of renal lesions using contrast-enhanced ultrasound (CEUS); a 10-year retrospective European single-centre analysis. Eur Radiol 2018; 28: 4542-9. 
Figure legends:

\section{Figure 1. Volumetric T2 imaging for tumour anatomy}

20-month-old male. Palpable abdominal mass and a left renal mass on ultrasound

a) Axial T2 image. The intra-renal location of the tumour is demarcated by surrounding renal parenchyma (arrow). Abdominal blood vessels and adjacent organs can be delineated.

b) Minimum intensity projection coronal reconstruction ( $2 \mathrm{~mm}$ thickness) demonstrating the arterial anatomy of the left renal artery relative to the left renal mass. Signal void within the vessel indicates normal blood flow. The artery is stretched over the tumour but there is no arterial involvement.

c) Minimum intensity projection coronal reconstruction ( $2 \mathrm{~mm}$ thickness) demonstrating the venous anatomy of the left kidney. The left renal vein is displaced superiorly with no evidence of venous extension.

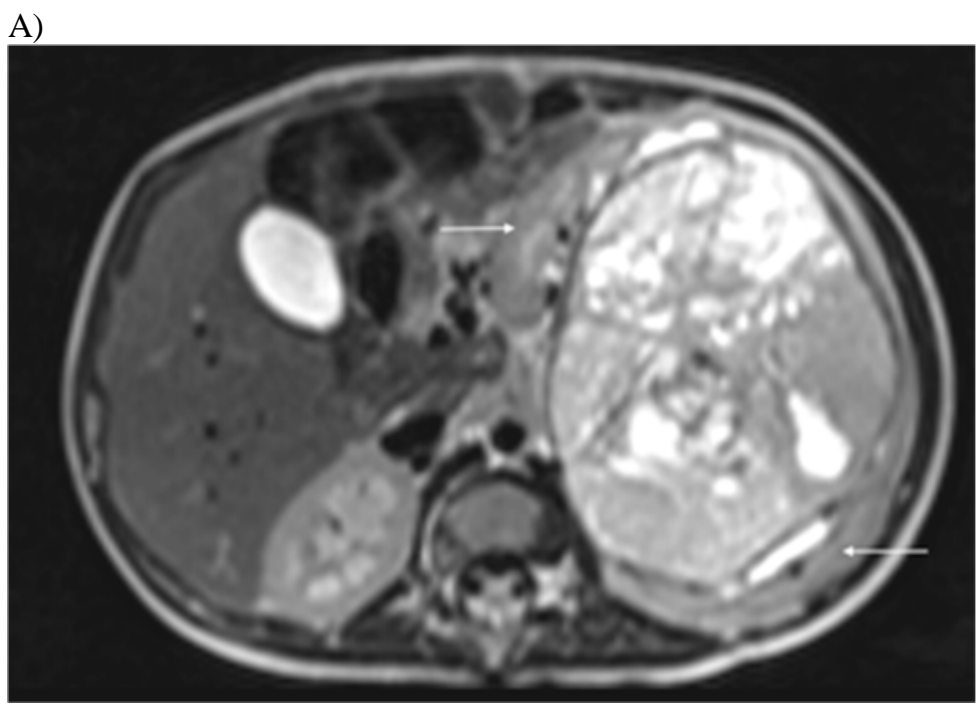

B)

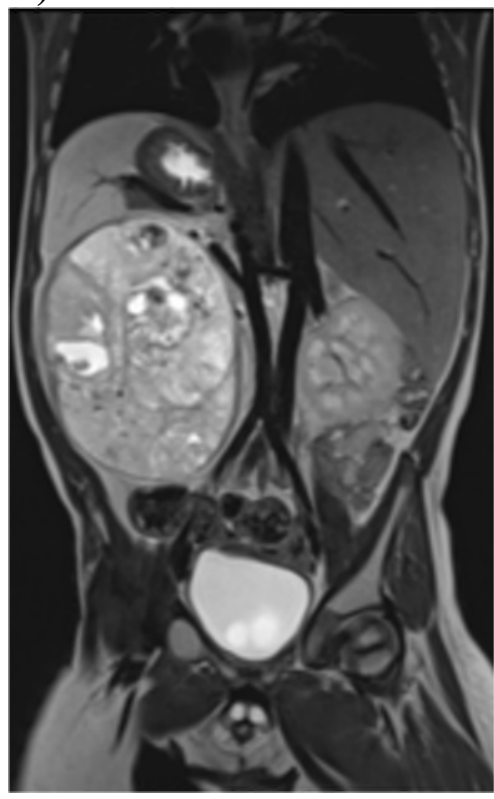


c)

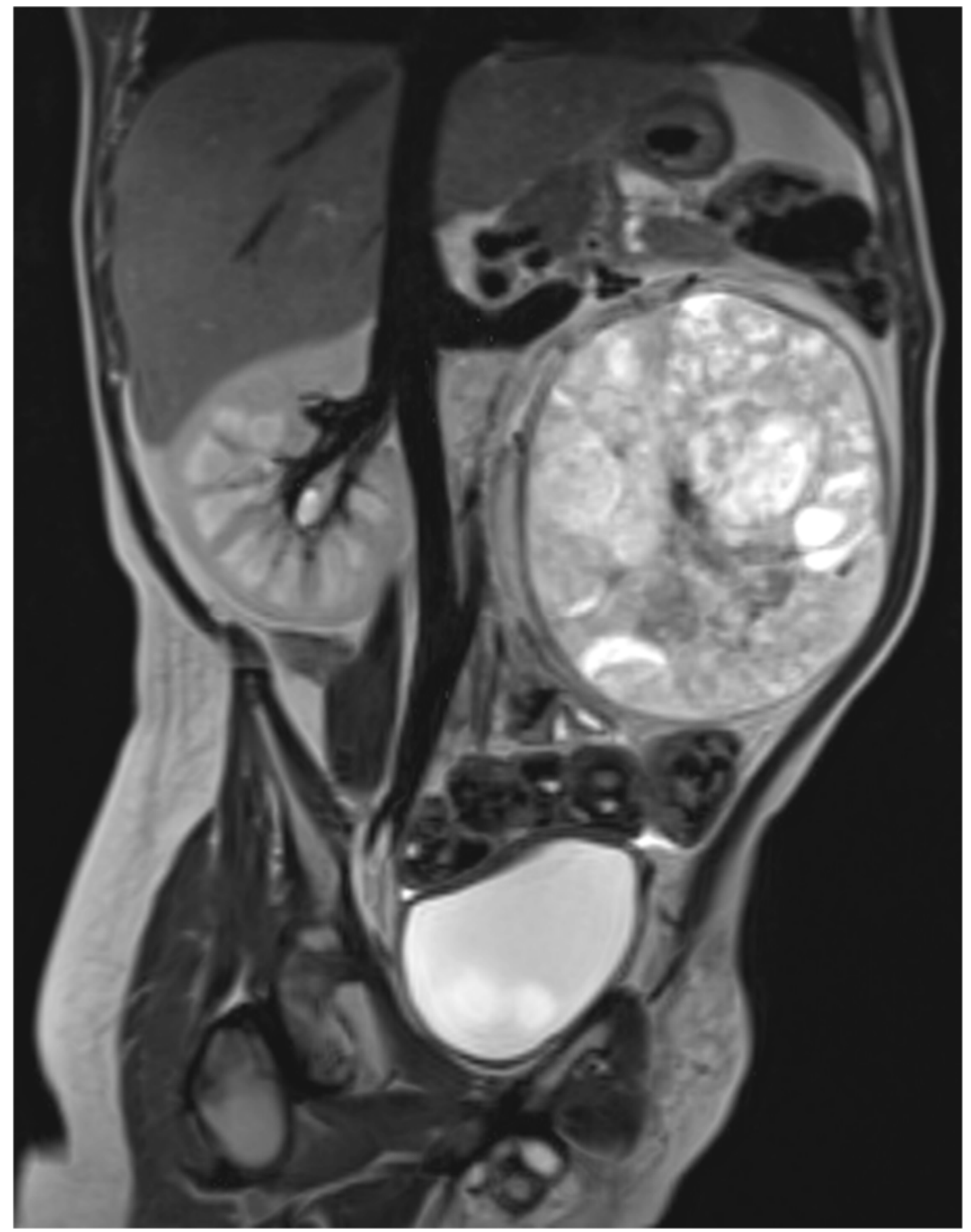




\section{Figure 2. Rupture of the tumour capsule}

3-year-old female presenting unwell with abdominal distension and a mass on ultrasound

a) Coronal T2 reconstruction demonstrates a large tumour arising from the right kidney. Exophytic part of the tumour extends into the subhepatic space associated with perinephric fluid (white arrows). Moderate ascites extends beyond the pelvic brim and further exophytic tumours are seen immediately inferior to the main renal mass (arrowhead).

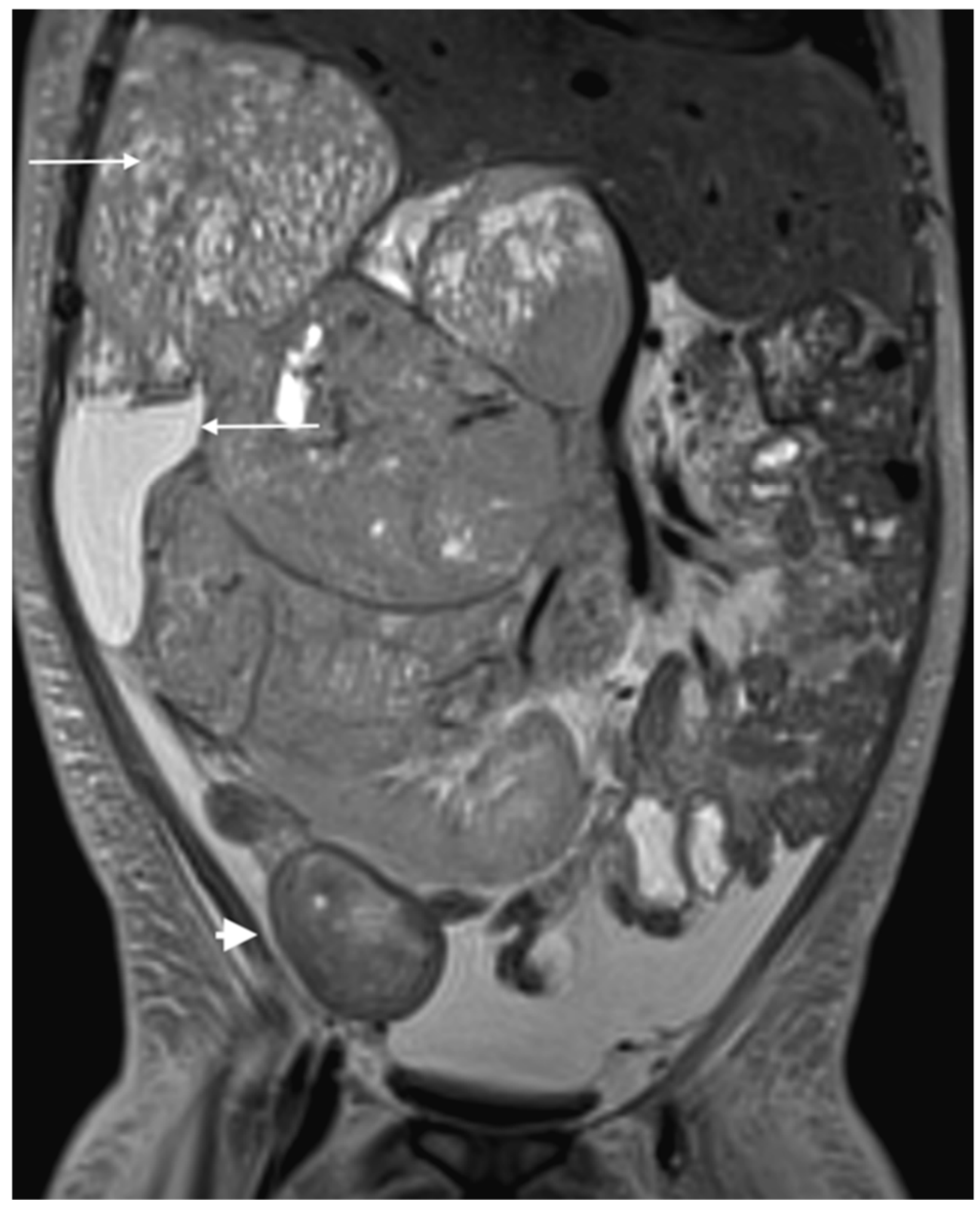


b) Sagittal T2 reconstruction demonstrates a fluid-fluid level within the ascites (arrow). This suggests blood product within the fluid, which is highly suspicious for intra-peritoneal tumour rupture (confirmed histologically).

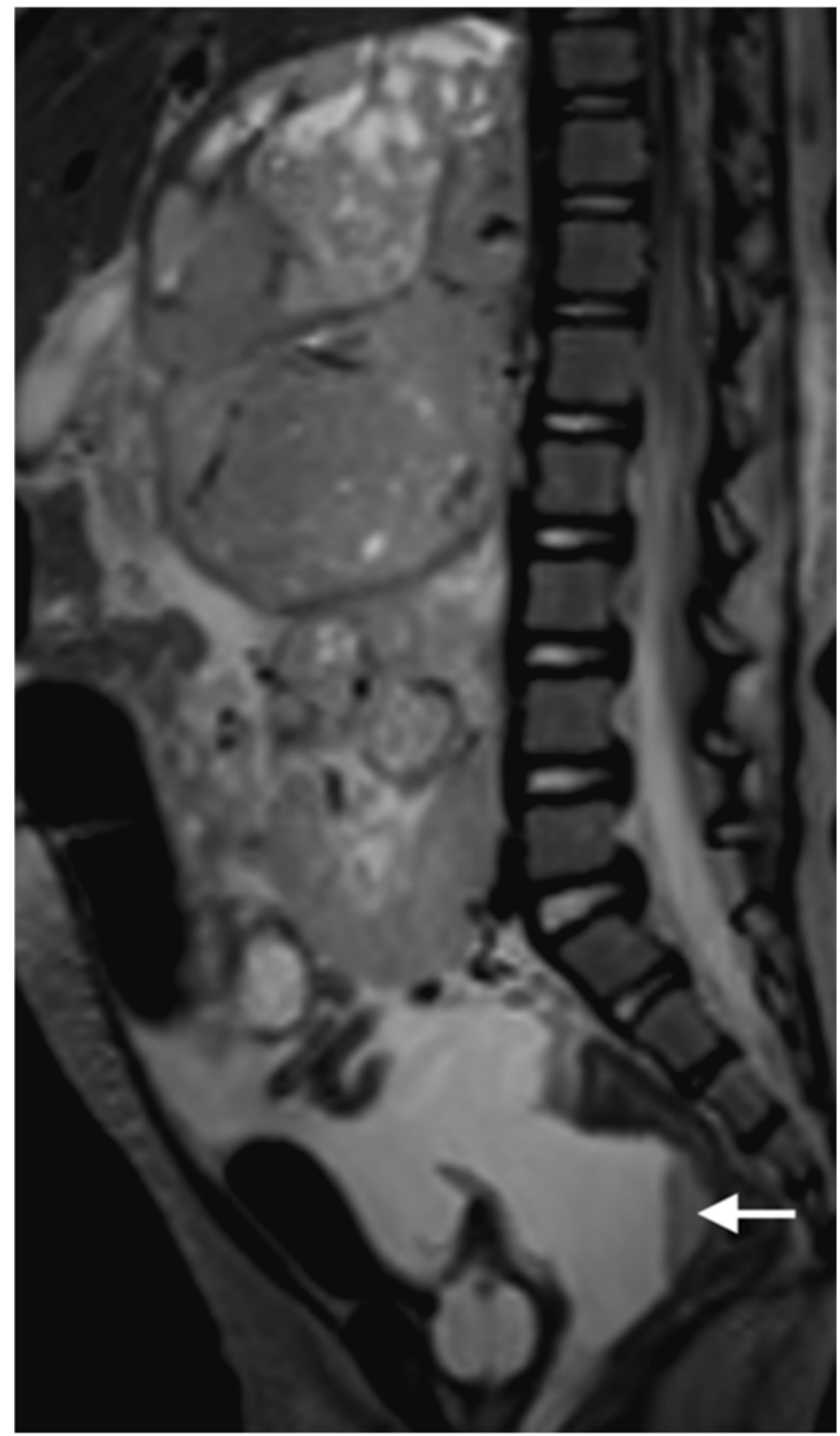

c)Axial T2 image. Extra-renal tumour displaces the aorta anteriorly (arrow). This may represent lymph node spread, but it is likely that this represents tumour rupture. A synchronous tumour is noted at the lower pole of the left kidney (arrowhead). 


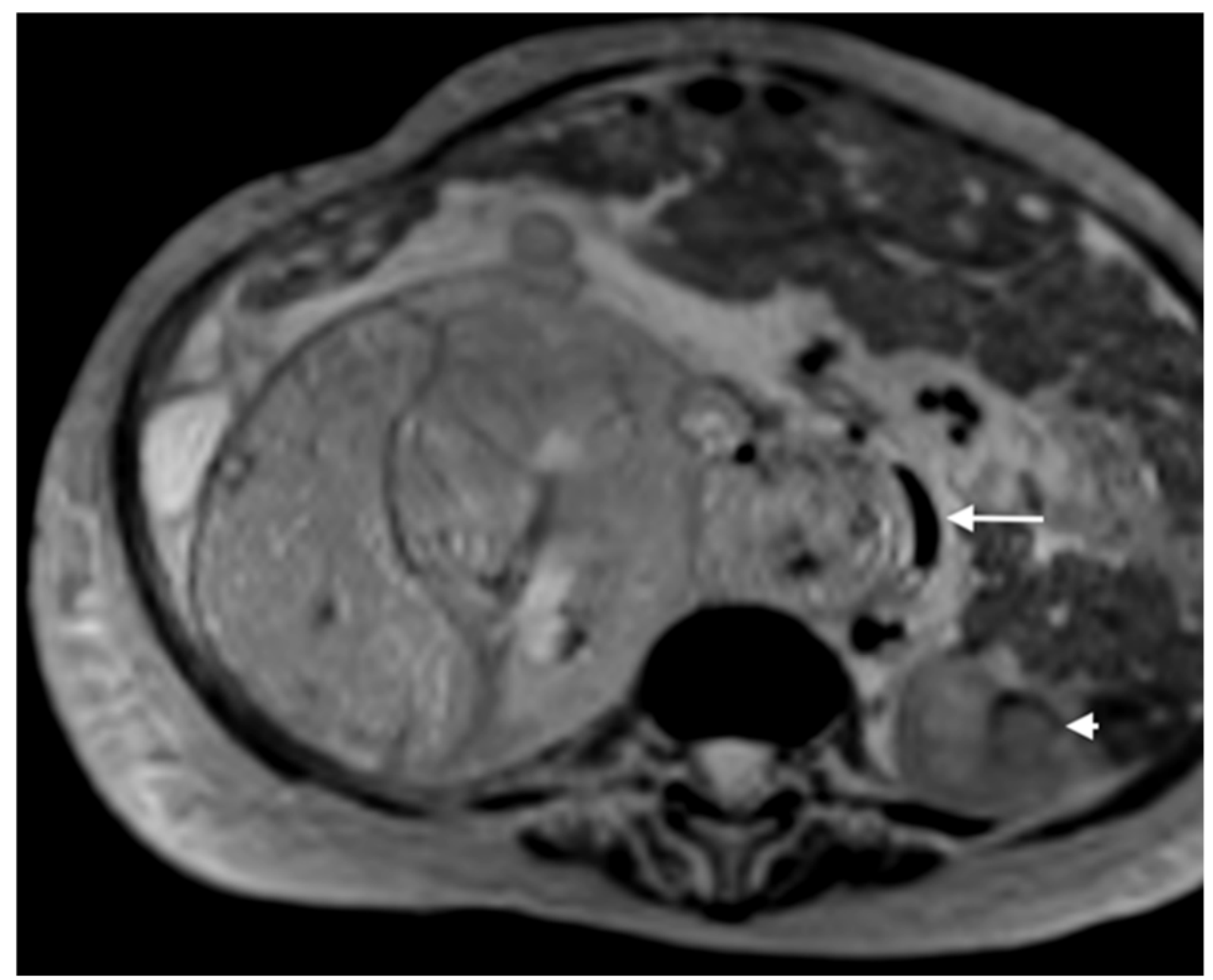




\section{Figure 3. invasion of the IVC due to tumour thrombus}

2-year-old female with an abdominal mass.

Coronal T2 reconstruction. Large heterogenous right-sided renal tumour with extension of tumour material into the IVC. The tumour thrombus extends as far as the IVC confluence and bulges into the right atrium which is just visible at the superior edge of the image (arrow).

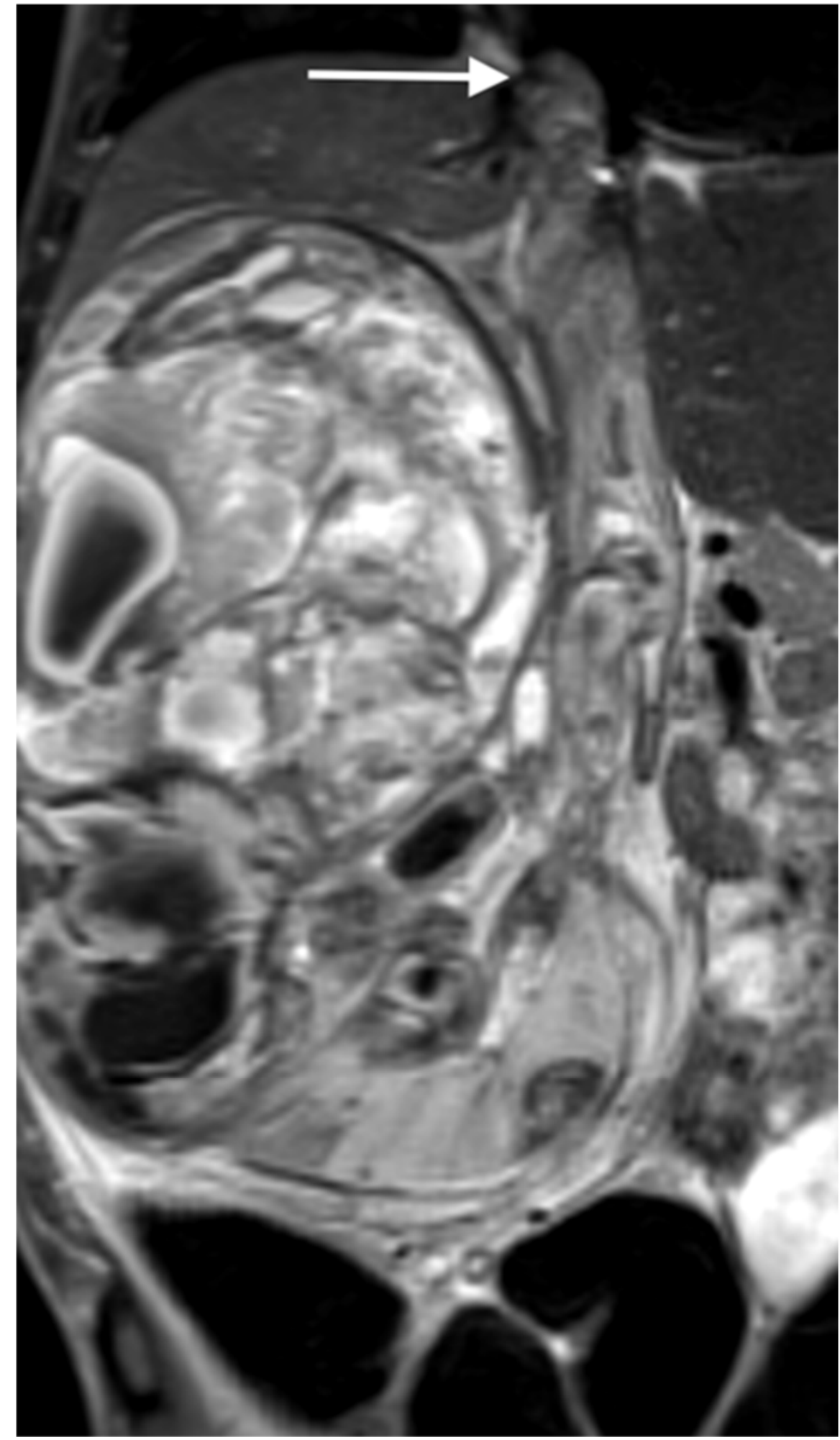




\section{Figure 4. Nephroblastomatosis before and after treatment}

18-month-old female. Abdominal distension.

a) Axial T2 pre-treatment image demonstrating large bilateral renal tumours. The majority of the normal renal parenchyma has been replaced.

b) Pre-treatment ADC map shows marked restriction of free water motion (arrows)within the tumours, normal renal parenchyma can be seen anteriorly on the left (arrowhead)

c) Axial T2 image 18 months after chemotherapy. The tumours have considerably reduced in size, but a rim of abnormal signal is seen surrounding both kidneys (arrows)

d) Corresponding Axial ADC map 18 months after treatment. The residual areas of disease still demonstrate restriction of free water motion.

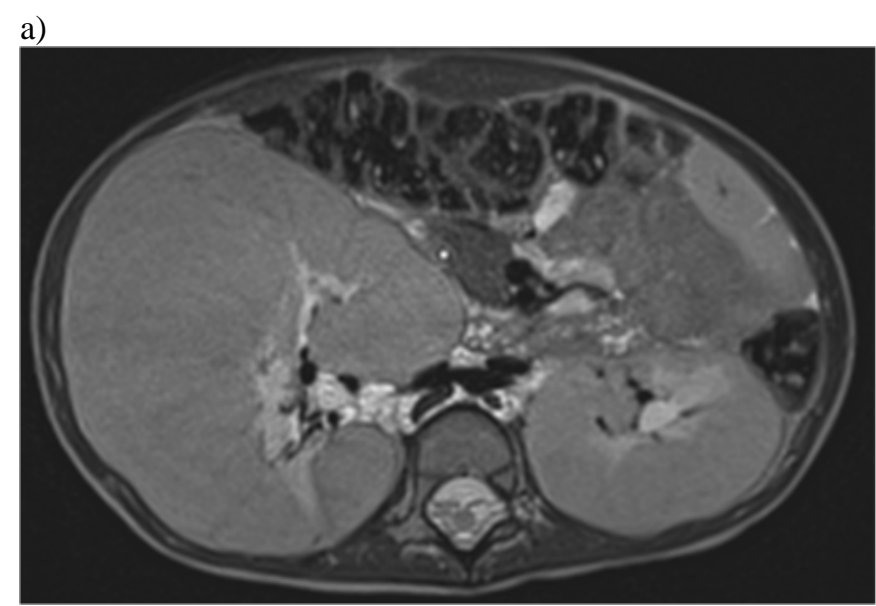

b)

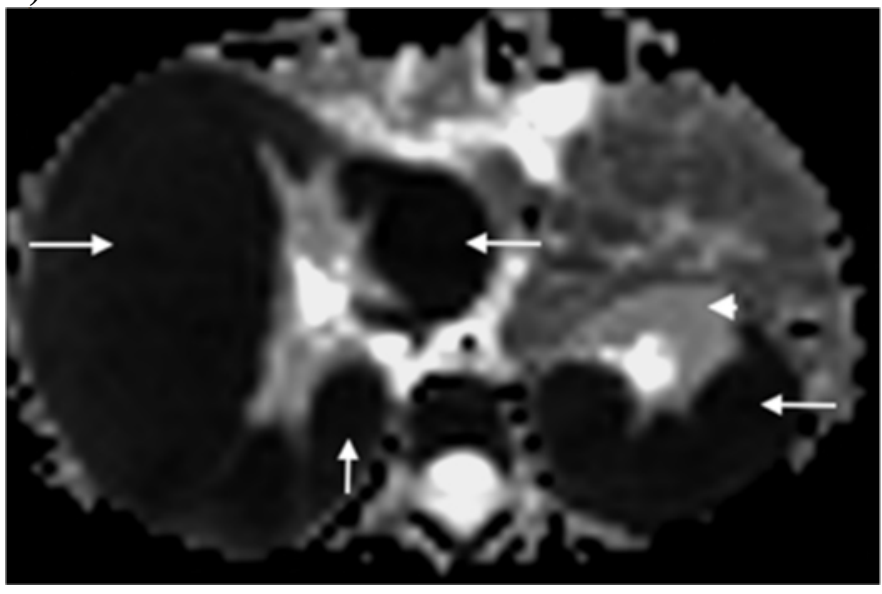




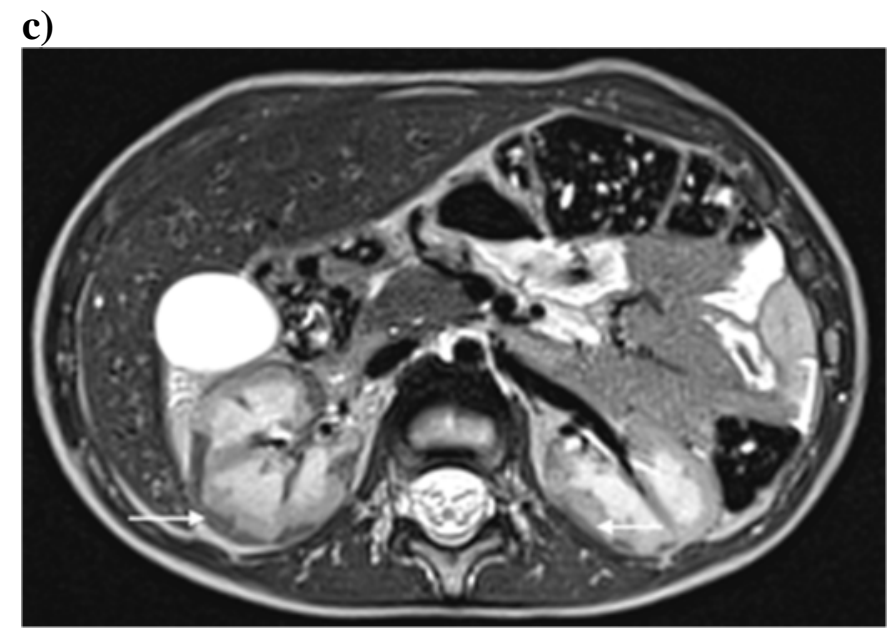

d)

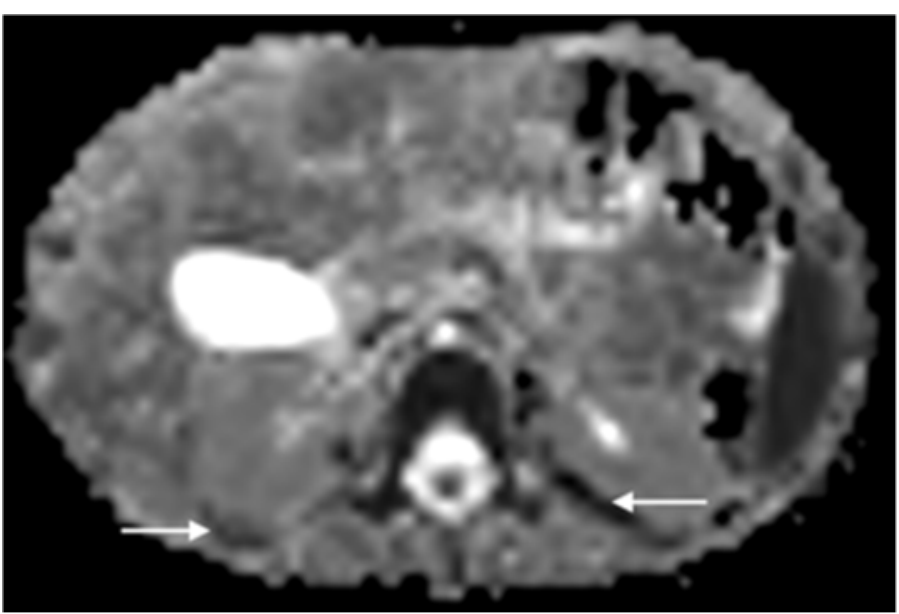




\section{Figure 5. Unencapsulated tumour}

5-year-old male with a large abdominal swelling.

(a) Axial T2 image shows a large tumour encasing the major vessels of the abdomen (arrows). Multiple lesions are also seen in the liver. Neuroblastoma was proven at biopsy.

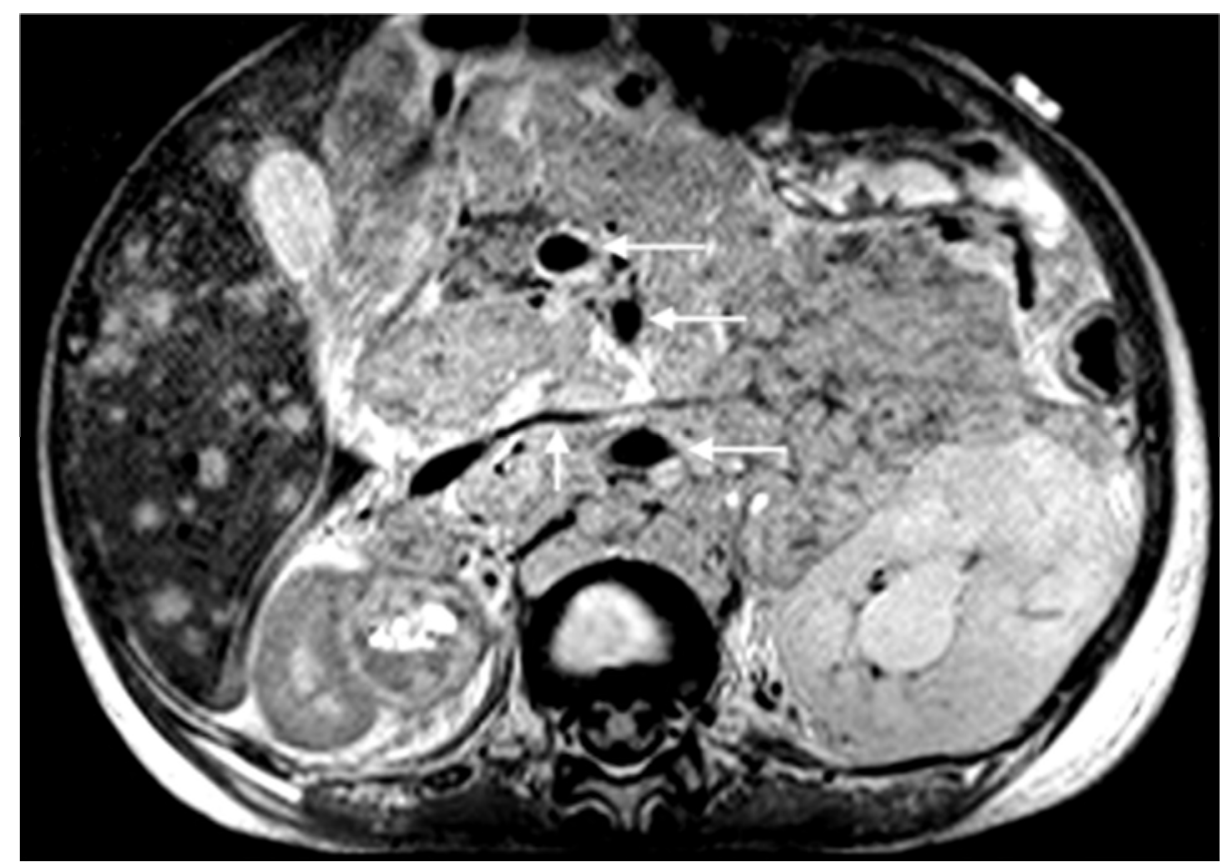

Large encapsulated mass arising from the left kidney in a 4-year-old male (b). The tumour is displacing the vessels of the abdomen (arrows) rather than encasing them. Wilms tumour was proven at histology.

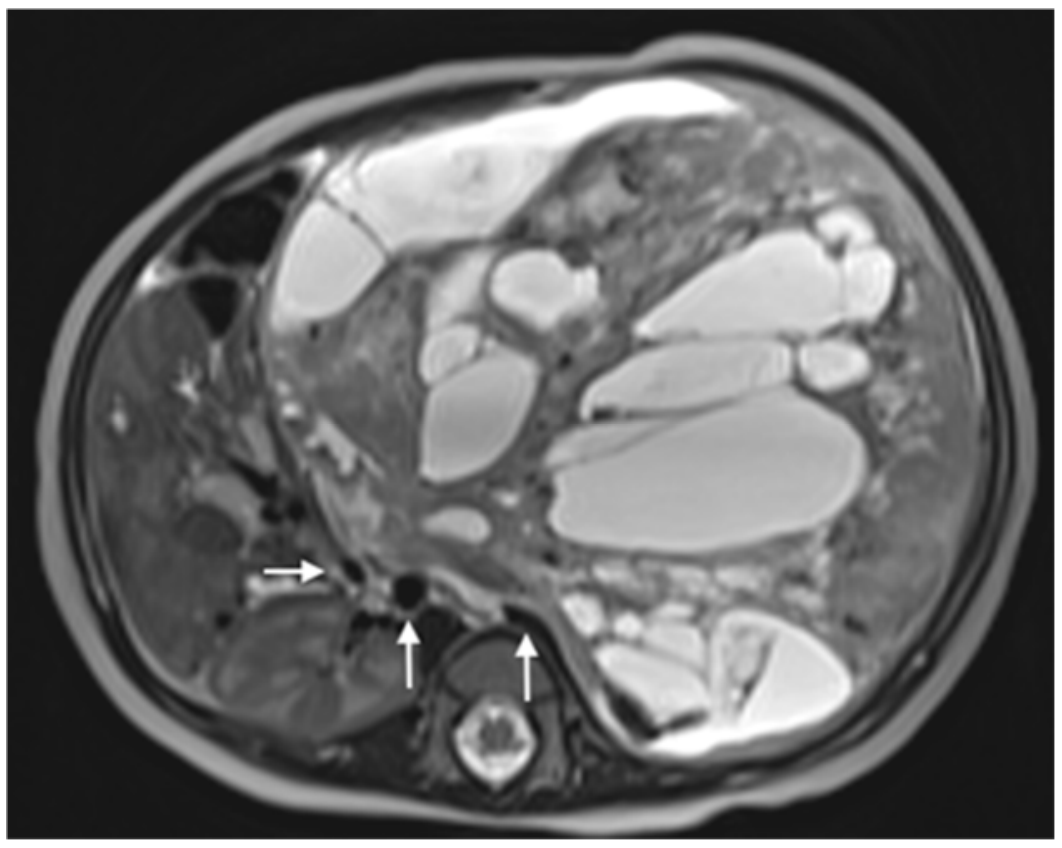




\section{Figure 6. Cystic Partially Differentiated Nephroma}

11-month-old male with a mass seen on a routine ultrasound performed for a urinary tract infection.

a) Axial T2 image demonstrates a completely cystic mass involving the left kidney.

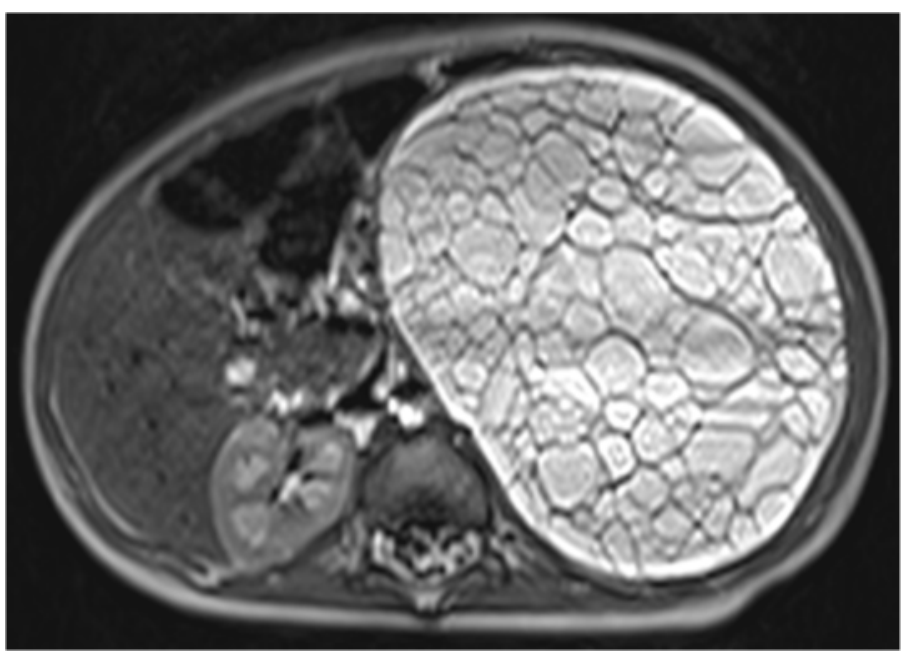

b) Axial ADC map of the same patient demonstrates no restriction of free water motion except in the septa between cysts.

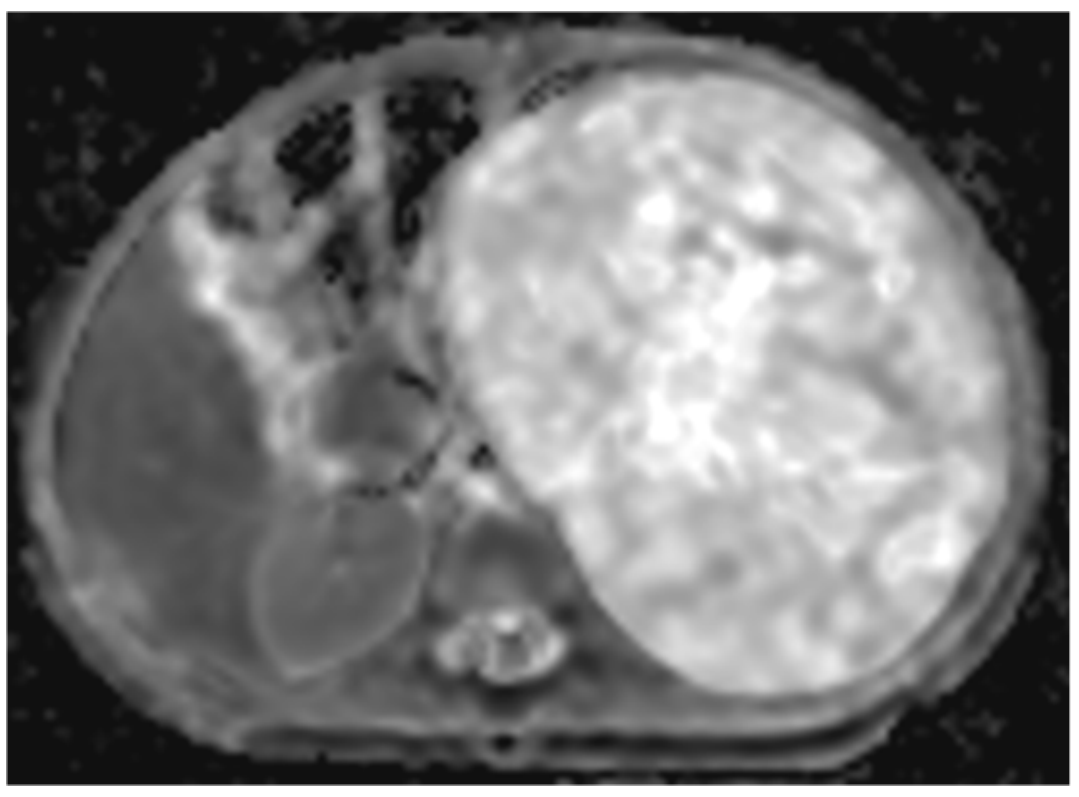


$6 \mathrm{c})$ Axial T1 image following administration of gadolinium. The tumour shows enhancement of the septi only, there are no large areas of solid tumour.

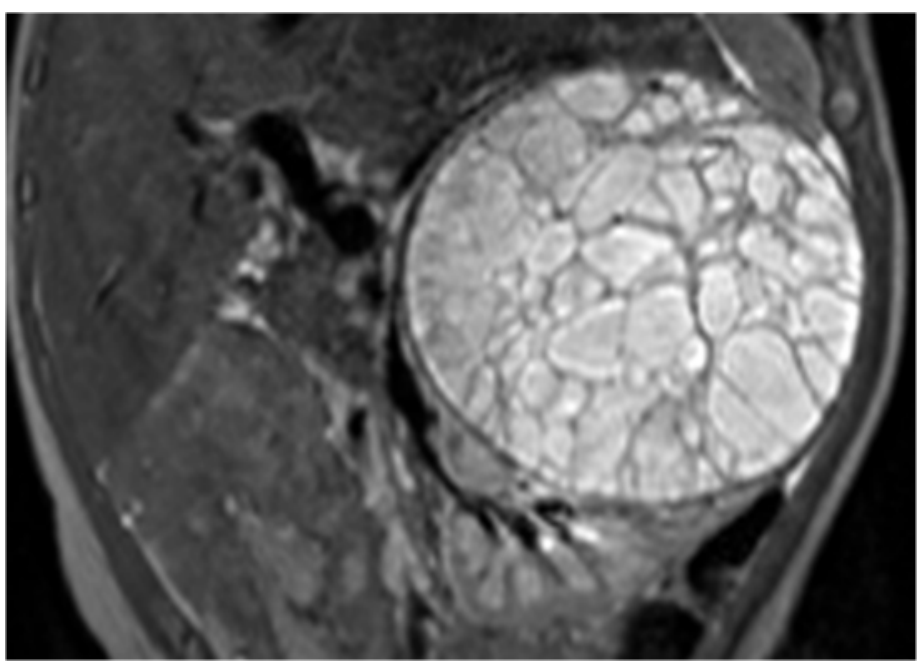




\section{Figure 7. Advanced DWI/ T1 mapping}

Axial slices from three Wilms tumours (outline in red). A, C, and $\mathbf{E}$ are generated using gadolinium. B, D, and $\mathbf{F}$ are the same Wilms tumours generated using $\mathrm{ADC}$ and $\mathrm{T}_{1} \mathrm{w}$ (without gadolinium). Increased signal represents more viable tissue. Tumour details: $\mathbf{A}$ and $\mathbf{B}$ - Mixed subtype, Age at Scan: 11 years, $\mathbf{C}$ and $\mathbf{D}-$

Blastemal subtype, Age at Scan: 1 year, $\mathbf{E}$ and $\mathbf{F}$ - Mixed subtype, Age at Scan: 1 year.

Reprinted from European Radiology, 29(8), 4141-4149. DOI: 10.1007/s00330-018-5907-z with authors permission, under the Creative Commons License https://creativecommons.org/licenses/by/4.0/

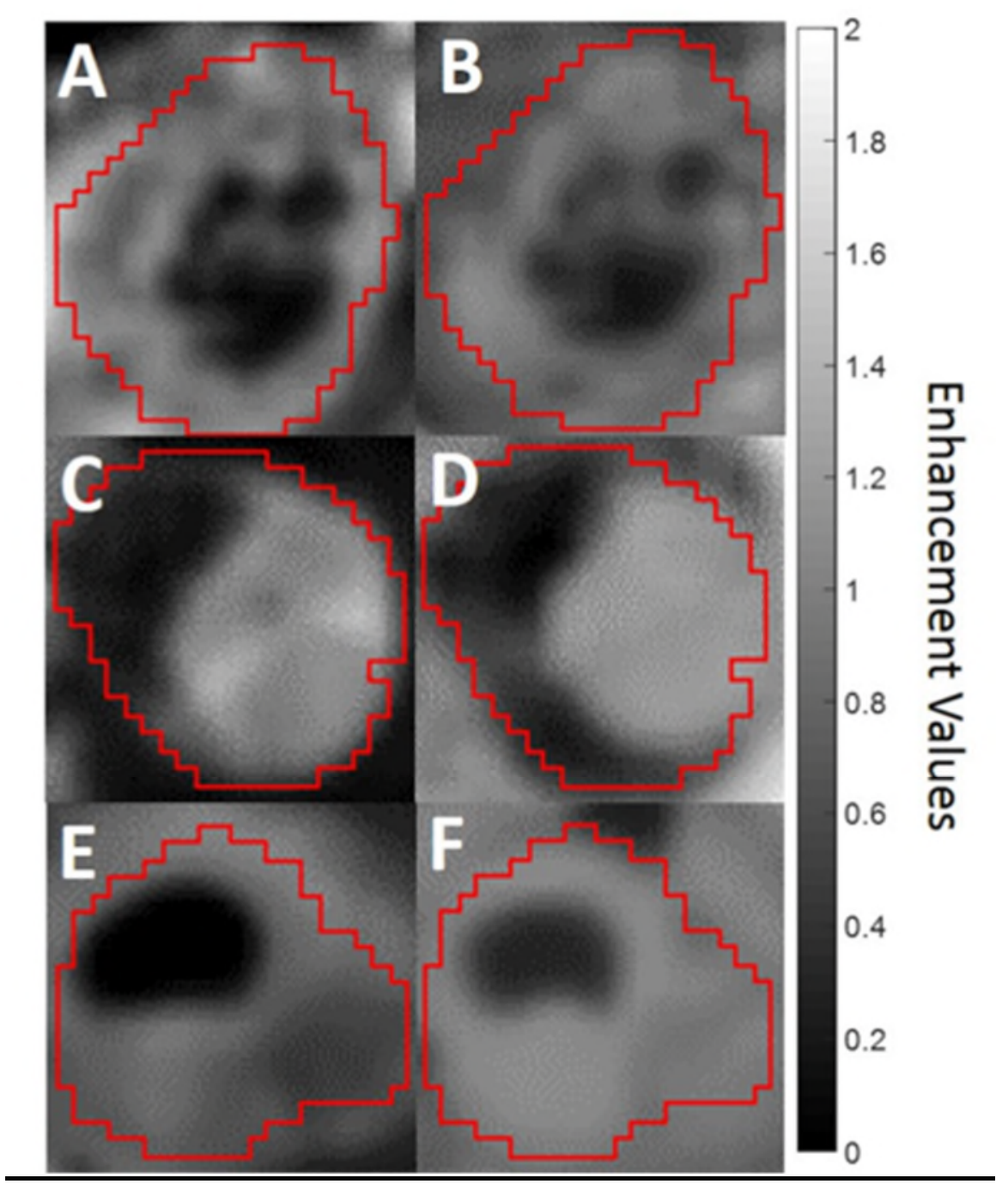


Table 1. Abdominal tumour protocol Great Ormond Street Hospital (GOSH).

\begin{tabular}{|c|c|c|c|c|c|}
\hline Parameter & T2W-STIR & T2-SPACE & DWI & T1 pre/post & T1 pre/post \\
\hline $\begin{array}{l}\text { Pulse } \\
\text { sequence }\end{array}$ & $\begin{array}{l}\text { 2-D short tau } \\
\text { inversion } \\
\text { recovery } \\
\text { (STIR) spin } \\
\text { echo }\end{array}$ & $\begin{array}{l}\text { 3-D turbo } \\
\text { spin-echo } \\
\text { with variable } \\
\text { flip angle }\end{array}$ & $\begin{array}{l}\text { 2-D single- } \\
\text { shot spin- } \\
\text { echo with } \\
\text { spectral fat } \\
\text { saturation }\end{array}$ & $\begin{array}{l}\text { 2-D turbo spin- } \\
\text { echo } \\
\text { with fat- } \\
\text { suppression }\end{array}$ & $\begin{array}{l}\text { 2-D turbo spin echo } \\
\text { with fat } \\
\text { suppression and } \\
\text { variable readout } \\
\text { directions }\end{array}$ \\
\hline TR (ms) & $>6,000$ & $>2,000$ & 2700 & $400-670$ & 590 \\
\hline TE (ms) & 62 & 238 & 90 & $17-20$ & 23 \\
\hline IR & 130 & & & & \\
\hline Orientation & Cor /Ax & $A x$ & $A x$ & $A x$ & $A x$ \\
\hline $\mathrm{ST}(\mathrm{mm})$ & $5-6$ & 0.9 & 6 & $5-6$ & 7 \\
\hline Gap (mm) & $0-0.5$ & 0 & $1.8-2.4$ & $1-3$ & 1.4 \\
\hline FOV & $250 \times 199$ & & $262 \times 350$ & $187 \times 250$ & $229 \times 229$ \\
\hline Acq matrix & $256 \times 194$ & $256 \times 255$ & $128 \times 96$ & $256 \times 154$ & $192 \times 192$ \\
\hline $\begin{array}{l}\text { Bandwidth } \\
\text { (Hz/pixel) }\end{array}$ & & & 1,500 & & \\
\hline $\begin{array}{l}\text { B-values } \\
\left(\mathrm{s} / \mathrm{mm}^{2}\right)\end{array}$ & - & - & $\begin{array}{l}0,50,100, \\
250,500 \\
1000\end{array}$ & - & - \\
\hline
\end{tabular}


Legend : Time to Repeat (TR) (ms), Time to Echo (TE), Inversion Recovery (IR), Coronal (Cor), Axial (Ax), Slice Thickness (ST) Field of View (FOV), Acquisition Matrix (Acq Matrix). 